\begin{tabular}{|c|c|c|}
\hline Tumor & No of cases & Percentage (\%) \\
\hline \multicolumn{3}{|l|}{ Breast } \\
\hline Known diagnosis & 12 & 21.4 \\
\hline New diagnosis & 4 & 7.1 \\
\hline \multicolumn{3}{|l|}{ New Unknown Primary } \\
\hline Metastatic carcinoma & 5 & 8.9 \\
\hline Adenocarcinoma, likely Gl origin & 3 & 5.4 \\
\hline Neuroendocrine carcinoma & 2 & 3.6 \\
\hline Lung, non-small cell (autopsy dx) & 1 & 1.8 \\
\hline \multicolumn{3}{|l|}{ Ewing/PNET } \\
\hline New diagnosis & 4 & 7.1 \\
\hline Known diagnosis & 2 & 3.6 \\
\hline \multicolumn{3}{|l|}{ Lung } \\
\hline Small cell carcinoma & 4 & 7.1 \\
\hline Non-small cell carcinoma & 1 & 1.8 \\
\hline \multicolumn{3}{|l|}{ Gastrointestinal/Hepatobiliary } \\
\hline $\mathrm{H} / \mathrm{OHCC}$ and colon (likely $\mathrm{HCC}$ ) & 1 & 1.8 \\
\hline Stomach, adenocarcinoma & 1 & 1.8 \\
\hline Esophageal, adenocarcinoma & 1 & 1.8 \\
\hline Colon, adenocarcinoma & 1 & 1.8 \\
\hline Gallbladder, small cell carcinoma & 1 & 1.8 \\
\hline \multicolumn{3}{|l|}{ Melanoma } \\
\hline Known diagnosis & 3 & 5.4 \\
\hline New diagnosis & 1 & 1.8 \\
\hline \multicolumn{3}{|l|}{ Prostate } \\
\hline Known diagnosis & 2 & 3.6 \\
\hline New diagnosis & 2 & 3.6 \\
\hline \multicolumn{3}{|l|}{ Miscellaneous Known Diagnoses } \\
\hline Transitional cell carcinoma & 1 & 1.8 \\
\hline Oligodendroglioma, anaplastic & 1 & 1.8 \\
\hline Merkel cell carcinoma & 1 & 1.8 \\
\hline \multicolumn{3}{|l|}{ Miscellaneous New Diagnoses } \\
\hline Favor renal cell carcinoma & 1 & 1.8 \\
\hline Angiosacroma & 1 & 1.8 \\
\hline
\end{tabular}

Median time from initial cancer diagnosis to BM biopsy was 1 month, and from biopsy to death was 3 months. BM biopsies showed extensive tumor burden (median $75 \%$, range $<5 \%-100 \%$ ), but percent replacement did not predict survival. Patients had elevated alkaline phosphatase (mean $373 \mathrm{U} / \mathrm{L}$ ) and lactate dehydrogenase (mean 1045 $\mathrm{U} / \mathrm{L})$, but only $\mathrm{LDH}$ was associated with more extensive $\mathrm{BM}$ involvement $\left(\mathrm{R}^{2}=0.13\right.$, $\mathrm{P}<0.05)$. Cytopenic patients $(\mathrm{n}=47)$ most frequently had thrombocytopenia $(\mathrm{n}=33)$, which correlated with shorter survival $\left(\mathrm{R}^{2}=0.10, \mathrm{P}<0.02\right)$ and more extensive $\mathrm{BM}$ involvement $\left(\mathrm{R}^{2}=0.12, \mathrm{P}<0.01\right)$. Patients frequently complained of musculoskeletal pain $(n=29)$, such as severe bone/back pain. Constitutional symptoms (e.g., fevers, chills, weight loss) and symptoms consistent with anemia (e.g., fatigue, dyspnea, weakness) were common $(n=27)$, as was radiologic evidence of bone disease $(n=13)$. With the exception of a patient with sacral mass (Ewing/PNET) and another with melanoma, new cancer patients presented with nonspecific symptoms $(n=15)$, musculoskeletal pain $(n=13)$, and bone lesions $(n=6)$.

Conclusions: In this study on metastatic cancer to the BM, musculoskeletal pain and nonspecific symptoms were common. Extensive BM replacement correlated with elevated LDH and thrombocytopenia, and the latter predicted worse outcomes. Even in the modern era, patients' initial presentation may be BM metastasis; therefore, we recommend diligently searching even routine BM biopsies for metastatic cancer.

1600 Clinicopathologic Features of Patients with Post-Transplant Lymphoproliferative Disorder (PTLD) Following Hematopoietic Stem Cell Transplant (HSCT) Are Different from PTLD in Solid organ Recipients (SOT)

$J$ Zhou, K Van Besien, A Orazi, E Cesarman, DM Knowles, JT Geyer. Weill Cornell Medical College, New York, NY.

Background: The clinical field of allogeneic HSCT and SOT is rapidly evolving with increasing use of cord blood, mismatched unrelated donors, T-cell depleted grafts and anti-T cell antibody therapy-all known risk factors for development of PTLD. Most large pathology studies of PTLD are found in the older literature prior to widespread use of allogeneic HSCT. The aim of this study was to compare the clinicopathologic features of PTLD following HSCT and SOT and to evaluate the effect of current transplantation practices.

Design: 26 biopsies from 21 patients diagnosed at our institution between 2002 and 2013 were available for review: 10 patients (48\%) had PTLD following HSCT (PTLD-HSCT: 5 allo-HSCT; 5 haplo/cord blood-HSCT) and 11 patients (52\%) had PTLD following SOT (PTLD-SOT: kidney or liver). Clinical, pathologic, cytogenetic and molecular features were assessed. Statistical analysis was performed.

Results: Patients with PTLD-HSCT had extranodal (5), nodal (3) or peripheral blood (2) involvement. $5 / 10$ patients were conditioned with ATG. Median interval between HSCT and PTLD was 3.5 (range 1.5-34) months. Based on WHO classification, 2 biopsies (14\%) were diagnosed as early lesion; 8 (57\%) had polymorphic PTLD (PTLD-P); 4 (29\%) had monomorphic PTLD (PTLD-M; 3 DLBCL and 1 T-LGL). EBV was positive in $9 / 10$ patients $(90 \%)$ ). $6 / 10$ patients died after a median of 2 (range, $0-9$ ) months after PTLD diagnosis. Patients with PTLD-SOT had extranodal (7) or nodal (4) involvement. Median interval between SOT and PTLD was 5 (range $0.75-16$ ) years. One patient $(9 \%)$ was diagnosed as PTLD-P; 9 (82\%) had PTLD-M (8 DLBCL; 1 Burkitt lymphoma); and $1(9 \%)$ had Hodgkin lymphoma. PTLD-M following SOT contained significantly more neoplastic B-cells than PTLD-M after HSCT $(\mathrm{p}<0.01)$. EBV was positive in $8 / 11$ patients (73\%). 3/10 patients died (median, 5; range, 0-36 months after PTLD diagnosis). In both groups, only PTLD-M had an abnormal karyotype. 8/10 (80\%) cases of PTLD-P and all PTLD-M had evidence of clonal IgH gene rearrangement. 4/7 (57\%) cases of PTLD-P also had oligoclonal or faint clonal T cell receptor gene rearrangements. Conclusions: PTLD-HSCT occur significantly sooner, have a higher proportion of PTLD-P, more polymorphous-appearing PTLD-M, are more frequently EBV+ and have a worse outcome $(\mathrm{p}<0.01)$. These differences may be due to different pathogenetic mechanisms, since PTLD-HSCT is typically donor-derived, while PTLD-SOT is of host origin. More studies are needed to explore these differences.
1601 High-Resolution SNP Array Analysis of B-Lymphoblastic Lymphoma Using Tissue from Archived Glass Slides Reveals a Genetic Profile That Differs from B-Lymphoblastic Leukemia

$D$ Zhou, C Christensen, V Rodic, JM Downie, AM Termuhlen, MA Lones, $M$ Abromowitch, SL Perkins, JD Schiffman, RR Miles. University of Utah Health Sciences Center, Salt Lake City, UT; Keck School of Medicine, University of Southern California, Los Angeles, CA; University of Nebraska Medical Center, Omaha, NE.

Background: Gene copy number change is a common feature in B-lymphoblastic leukemia (B-ALL), and abnormal copy number of certain genes, such as $I K Z F 1$, is associated with prognosis. B-lymphoblastic lymphoma (B-LBL) is closely related to B-ALL but presents with no or minimal bone marrow involvement. Unlike B-ALL, gene copy number analysis of B-LBL has been limited by the lack of fresh or frozen tissue for genomic testing. Molecular inversion probe (MIP)-based SNP array is an effective approach for high-resolution copy number analysis of formalin-fixed, paraffin-embedded (FFPE) archival tissues. In the present study, we analyzed FFPE B-LBL specimens using tissue scraped from glass slides and compared the results to previous studies of B-ALL. Design: Unstained FFPE slides from the COG A5971 trial were available for B-LBL cases $(\mathrm{N}=16$; Median age $=6$ years; Male:Female $=7: 9)$. Slides have been stored at room temperature for 8-10 years. DNA was extracted from 9-10 sections per specimen and analyzed on the Affymetrix OncoScan FFPE Express 2.0 platform, a SNP array utilizing MIP technology. Data were analyzed using Nexus Copy Number software (BioDiscovery).

Results: Genome-wide copy number data were generated from 16/16 cases, and 15/16 cases showed at least 1 gain or loss $(93.8 \%)$. The median number of gains and losses per case was 3 (range $0-9$ ) and 1 (range $0-5$ ), respectively. Similar to B-ALL, CDKN2A deletions were identified in $4 / 16(25 \%)$, and hyperdiploidy involving trisomies of $4,6,8$, $10,11,17,18,21$ and $\mathrm{X}$ was observed in $5 / 16(31.3 \%)$. We found two features that differ from B-ALL: 1) B-cell development gene deletions (1 IKZF1, 2 PAX5) were observed in 3/16 (18.8\%), which is lower than reported in B-ALL (48.3\%); 2) Immunoglobulin light chain lambda (IGL) locus deletions that extend to the $\mathrm{VJ}$ junction, consistent with normal light chain rearrangement, were observed in $4 / 16$ (25\%), compared to only $1 \%$ in B-ALL. None of the B-LBL cases showed abnormal, focal IGL deletions, which we have recently identified in 18\% of B-ALL (Leukemia, Epub 7/24/13).

Conclusions: Our study demonstrates the utility of MIP array analysis for the study of archived samples of rare tumors. Our results show that the gene copy number profile of B-LBL has distinct differences from B-ALL suggesting possible differences in pathogenesis between these closely related diseases.

\section{Infectious Disease Pathology}

1602 Splenic Granulomas: Where Do They All Come From?

OI Ahmed, SA Qasem. Wake Forest School of Medicine, Winston Salem, NC. Background: There are several indications for surgical removal of the spleen including trauma, hematologic malignancies, cytopenias, iatrogenic injury, for diagnostic purposes and as part of another surgical procedure (e.g. pancreatectomy). Most commonly, the histology of the spleen is unremarkable; however, occasionally the surgical pathologist encounters granulomas in the spleen as an incidental finding. What are the different types of granulomas in the spleen; what are the etiologic factors behind them; and how often are they associated with systemic diseases?

Design: To answer these questions we reviewed our pathology database for all cases with granuloma in the spleen since 1990 . We classified granulomas into four major categories: necrotizing/caseating/suppurative (NCS) granulomas; non-necrotizing/noncaseating (NN) granulomas; hyalinized/calcified (HC) granulomas; and lipogranulomas (LG). Additionally, we collected the results of special stains (AFB, PAS, GMS), and reviewed the clinical records for associated systemic diseases and other organs involved. Results: Our search retrieved 131 cases (3\%) of splenic granulomas among 3932 spleen specimens; these included 106 surgical cases, 22 autopsies and 3 cytology specimens. The granulomas included $40 \mathrm{NN}(31 \%), 17 \mathrm{NCS}(13 \%), 16 \mathrm{HC}(12 \%)$ and $57 \mathrm{LG}(44 \%)$. Special stains were available for 39 cases. Among non-lipogranulomas, an infectious etiology was detected in 13 cases (18\%). Infectious agents included 8 fungi, 4 mycobacteria and 1 bacteria. Not surprisingly, NCS granulomas showed the highest rate of positivity for infection (53\%). On the other hand, systemic involvement was more common in NN cases $(48 \%)$ which also showed significant association with malignant neoplasms (25\%) and sarcoidosis (12\%).

Table 1

\begin{tabular}{|c|c|c|c|c|c|c|c|}
\hline Types & No. & Stained & Pos. stains & Infection & MAL & SAR & Other organs \\
\hline $\mathrm{NN}$ & 40 & 19 & 2 & 3 & 10 & 5 & 19 \\
\hline NCS & 17 & 14 & $\sqrt{7}$ & 9 & 5 & 0 & 5 \\
\hline $\mathrm{HC}$ & 16 & 4 & 1 & 1 & 2 & 0 & $\sqrt{4}$ \\
\hline LG & 57 & 1 & 0 & 0 & 11 & 0 & 0 \\
\hline Other & 1 & 1 & 0 & 0 & 1 & 0 & 0 \\
\hline All & 131 & 39 & 10 & 13 & 29 & 5 & 28 \\
\hline
\end{tabular}

MAL: malignancy; SAR: sarcoidosis; Pos: positive.

Conclusions: Granulomatous inflammation in the spleen is discovered incidentally in the majority of cases and can be associated with infectious, neoplastic or inflammatory conditions. Lipogranulomas are the most common type and their clinical significance is not clear. NCS granulomas have a strong association with mycobacterial and fungal infections, whereas NN are often associated with systemic diseases. It is important to recognize the different type of granulomas in the spleen and pursue additional studies, when appropriate. 
1603 qPCR Using Formalin-Fixed, Paraffin-Embedded Tissue Is a Highly Sensitive, Highly Specific Diagnostic Adjunct to H\&E Microscopy in the Detection of Giardia Spp. in Small Intestinal Biopsy Specimens

E Choi, Z Zhao, M McCoy, A Thomasson, J Lin. Indiana University School of Medicine, Indianapolis, IN.

Background: The diagnosis of Giardia spp. may pose a diagnostic challenge on routine hematoxylin and eosin (H\&E) sections of small intestinal biopsy specimens. Additionally, clinicians may not order additional laboratory testing for Giardia at the time of the biopsy due to the frequently nonspecific clinical presentation. We selected cases diagnosed as positive for Giarda by H\&E microscopy and tested the corresponding formalin-fixed, paraffin-embedded (FFPE) tissue using a quantitative real-time polymerase chain reaction (qPCR) to evaluate the performance characteristics of qPCR in detecting Giardia.

Design: We obtained 65 small intestinal biopsy specimens from the years 1991-2012 that were diagnosed as positive for Giardia on H\&E sections. We then tested the cases for evidence of Giardia by qPCR using the FFPE tissue. Seventy negative control biopsies from the small intestine and colon were also tested. A single case diagnosed as equivocal for Giardia by H\&E microscopy was also selected. A review of the medical records for the positive cases was done to determine whether any additional laboratory testing for Giardia spp. had been performed at the time of the biopsy.

Results: Of the 65 biopsy specimens positive for Giardia by H\&E, 64 also tested positive by qPCR, resulting in a sensitivity of $98.5 \%$. All 70 negative control cases were also negative by qPCR, resulting in a specificity of $100 \%$. The equivocal case tested negative for Giardia by qPCR. Pertinent medical records were available for 21 of the Giardia positive cases. Of these, only one case had a corresponding stool ova and parasite examination, which was also positive for Giardia. The remaining 20 cases did not have any additional laboratory testing peformed for Giardia at the time of the biopsy. Conclusions: qPCR using FFPE tissue is a useful, highly sensitive and highly specific molecular adjunct to H\&E histology in the diagnosis of Giardia, especially in equivocal cases. qPCR may help facilitate an accurate diagnosis in cases with nonspecific clinical presentations for which an endoscopic examination with biopsy is performed.

1604 Correlation of Fine-Needle Aspiration Cytology with Microbiologic Culture: A 14-Year Experience at a Single Institution

C Clement, R Nawgiri, $N$ Williams-Bouyer, V Schnadig. University of Texas Medical Branch, Galveston, TX

Background: Fine needle aspiration (FNA) is an important tool for infectious disease diagnosis. An aliquot of the FNA material should be submitted for culture confirmation following rapid on-site identification of microorganisms or inflammatory reactions. At our institution, correlation of FNA diagnosis with culture results and communication with Microbiology Laboratory personnel is common and considered important for patient care, education and quality control purposes. This study reviews 14 years of FNA-culture correlation

Design: FNA-culture correlation records were retrieved from FNA-histology-culture databases from 1996-2007 and 2010-2011. FNA-culture correlation was limited to cases with cultured material submitted directly from the FNA sample. At least one special stain was performed on most FNA in which microorganisms were identified. Cases with microorganisms identified by cytology, culture or cytology \& culture were categorized as: Bacteria, Actinomycetales, Mycobacteria or Fungi. Actinomycetales category includes Actinomyces, Nocardia and Rhodococcus. Mycobacteria category includes Mycobacterium spp. Culture is routinely done whenever purulent or granulomatous inflammation is found on rapid stain.

Results: Culture and FNA results from 686 samples were correlated. Cytology, culture or both were positive in 374/686 (55\%) samples excluding cultured bacterial skin contaminants. Bacteria (185): Cytology only positive $19(10 \%)$, cytology \& culture positive $84(45 \%)$, culture only positive $82(45 \%)$. Actinomycetales (15): Cytology only $3(20 \%)$, cytology \& culture 7 (47\%), culture only $5(33 \%)$. Mycobacteria 112 : Cytology only $19(17 \%)$, cytology\& culture $51(46 \%)$, culture only $42(37 \%)$. Fungi (62): Cytology only $30(49 \%)$ with $14 / 30$ being hyphal fungi, cytology \& culture 25 $(40 \%)$ with $100 \%$ concordance, culture only $7(11 \%)$

Conclusions: Correlation of cytopathology diagnoses with culture results and close communication with microbiology personnel is valuable for patient care and education. In 71 out of $374(19 \%)$ samples, organisms were identified only by cytology. Cytology only diagnoses were frequently fungi, especially hyphal forms. Cytology-microbiology correlation for yeast-like fungi was excellent. Monitoring of cytology-microbiology correlation is an important quality assurance tool.

1605 Synthesis and Implementation of Reference Plasmids for Use in the Clinical Pathology Laboratory

A Ishwar, C Hemphill, MP Alfaro, CJ Sailey. ACH, Little Rock, AR; UAMS, Little Rock, AR.

Background: There is a need for qualitative PCR standards as positive controls and quantitative standards for standard curves in clinical laboratories. Plasmid molecules carrying the target (or targets) of interest can fill this need. These can aid in validation and QC of real-time PCR (rtPCR) assays through measurement of sensitivity, specificity and limits of detection. Plasmids are simpler to control, measure, and preparations in clinical labs are cost effective and stable.

Design: PCR products for 14 organisms (B. pertussis, CMV, EBV, E. chaffeensis, HSV $1 \& 2$, K. kingae, S. pneumonia, Flu B, H3N2, HIN1 \& 2009 HIN1, S. aureus [Nuc \& $\mathrm{Mec} A]$ ) containing real-time targets were generated from DNA extracted from positive patient specimens using Taq polymerase, and target specific primers. For $B$. pertussis, a synthetic segment of the IS481 gene was used (Invitrogen). For influenza, a segment of hemagglutinin gene was used (IDT). All products were gel purified, ligated into pCR
2.1 vector and transformed into competent $E$. coli cells. Transformants were plated onto ampicillin agar with blue/white selection. Following colony PCR, positive plasmids were purified (QIAprep) and sequenced. To create a novel plasmid containing all targets, multiple smaller targets were fused using Overlap Extension PCR (OE-PCR). Primers were designed to generate fragments with overlapping complementary 3' ends. 2-3 fragments were mixed, followed by PCR in the presence of Phusion High-Fidelity DNA Polymerase (NEB) to generate the fused template, which was then further amplified. In cases where fusion failed, GC rich overlaps were utilized. Desired fragments were gel purified and cloned into pCR 2.1. A final plasmid was generated by step-wise cloning through restriction digestion and ligation.

Results: The final construct generated with the expected size of $11 \mathrm{~kb}$ was confirmed by electrophoresis. Sanger sequences confirmed each of the 14 targets and they were verified by rtPCR. Analytical and clinical sensitivity/specificity, reproducibility, and accuracy were confirmed for each. The 1:1 ratio of target to plasmid allows for quantitation using OD measurement without the need to extract from culture or use McFarland standards. LODs between 1-10 copies/uL have been measured and remain stable at $4^{\circ} \mathrm{C}$ over several months.

Conclusions: Plasmids for 14 bacterial/viral targets were created and combined into a final construct for use with rtPCR. These plasmids were created in the clinical laboratory and successfully implemented as controls, standards, and quality monitors for rtPCR, according to CAP/CLIA standards.

1606 Pathologic Diagnosis of Aspergillosis Revisited: Fungal Sequencing of Paraffin-Embedded Tissue Specimens for Identification

$Y$ Jiang, SL Rasor, JL Lovett, KL Muldrew. University of Toledo Medical Center, Toledo, $\mathrm{OH}$

Background: Aspergillus is a common fungal agent which can cause systemic as well as localized infection. Aspergillus has a morphologic feature of uniform 45 branching hyphae in tissue specimens, which is frequently used to indicate an infection by this agent. However, it is not definitive. The exact identity of fungal agents relies on a positive fungal culture. Fungal sequencing is a feasible method when using previously fixed, paraffin-embedded tissues. Here we compare the pathologic diagnosis of Aspergillosis to a lab developed fungal sequencing assay using paraffin-embedded tissue specimens. Design: Thirty four cases with a histopathologic diagnosis of fungal infection during the last 10 years were identified in our PowerPath database. Nineteen cases were suspicious for Aspergillosis by histopathologic examination. Among these, 9 cases with the presence of moderate to abundant amount of fungal hyphae in the specimen were used for DNA sequencing. These cases include 3 autopsy and 6 surgical pathology cases. DNA was extracted from paraffin-embedded tissue sections using Qiagen DNeasy Tissue Kit. Broad-range fungal primers were used to amplify a region of the rRNA genes using polymerase chain reaction (PCR). Cycle sequencing products were separated and identified using capillary electrophoresis. Sequences were then compared to a database (GenBank, NCBI) of sequences to determine the identification of the organism.

Results: Eight of the 9 cases gave acceptable sequence data for analysis. Among the 8 cases, only 4 cases were confirmed to have Aspergillus infection by fungal sequencing. In the other 4 cases, Pseudallescheria boydii, Alternaria sp, and Candida lusitaniae (2 cases) were identified as causative agents

Conclusions: Identifying the causative agents of fungal infection based on histomorphologic examination is difficult and not reliable due to overlapping of morphologic features. Fungal culture and fungal sequencing are two reliable diagnostic modalities with fungal sequencing especially useful in previously fixed and paraffin embedded tissues where a culture result is negative or a rapid diagnosis is needed.

\section{Cystoisospora Infection of the Gallbladder in Immunocompetent} Patients: A Clinicopathologic Review of 10 Cases

KK Lai, KA Miller, M Tuohy, GW Procop, LW Lamps, DT Patil. UAMS, Little Rock, AR; Cleveland Clinic, Cleveland, $\mathrm{OH}$.

Background: Cystoisospora (formerly Isospora) belli is an obligate intracellular coccidian that typically infects the small bowel in immunocompromised patients. A previous case series of patients with intestinal cystoisosporiasis found that nearly $15 \%$ had an increased alkaline phosphatase, suggesting that Cystoisospora infection of the biliary tree may be under-recognized as it has only rarely been reported. This study reports the clinicopathologic features of 10 cases of gallbladder cystoisosporiasis, the largest series to date.

Design: Ten cases over a 5 -year period were re-reviewed by 3 pathologists with infectious disease expertise. Clinicopathologic features were compared against a control group of 22 age and gender-matched patients with cholecystectomies.

Results: Cystoisospora was histologically confirmed by the identification of characteristic intraepithelial parasites within perinuclear, parasitophorous vacuoles with distribution ranging from focal to diffuse. Surface epithelial disarray was noted in $9 / 10$ cases. Intraepithelial lymphocytosis was present in $7 / 10$ cases with rare intraepithelial lymphocytes seen in the rest. No cases exhibited active inflammation, mucosal blunting or a prominent eosinophilic infiltrate. 


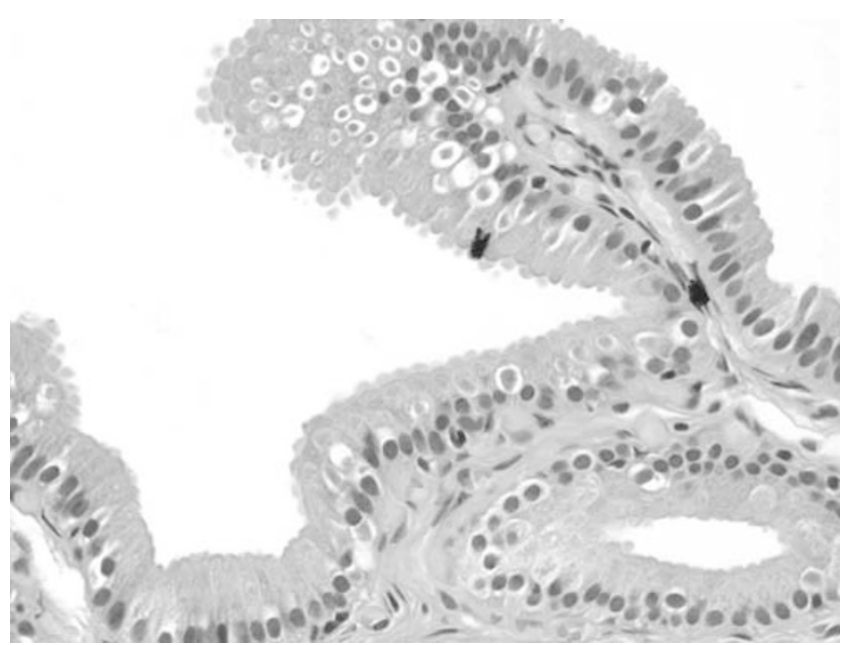

Of the 10 cases, 2 were incidentally identified in liver donors without additional clinical history. Mean age of the remaining 8 patients was 32 years with M:F ratio of 3:1. Six patients were immunocompetent, while immune status was unknown in two. Cholelithiasis, abdominal pain and dyskinesia were surgical indications in $25 \%$, $75 \%$ and $50 \%$ of cases, respectively. The control group had mean age of 40 years and M:F ratio of $1: 1$. Surgical indications included cholelithiasis, abdominal pain and dyskinesia in $59 \%, 73 \%$ and $9 \%$, respectively. Calculi were grossly identified in $1 / 10$ cases of cystoisosporiasis and $14 / 22$ controls. This difference was statistically significant $(\mathrm{p}=0.006)$.

Conclusions: This study confirms that Cystoisospora infection of the gallbladder is an under-recognized entity that may present incidentally or with symptomatic disease in immunocompetent patients. Unlike other sites, gallbladder cystoisosporiasis may not be associated with an eosinophilic infiltrate.

\section{Rapid, PCR-Based Identification of any Fungus from Culture}

DE Nowak, R Tibbetts. Henry Ford Hospital, Detroit, MI.

Background: The identification of fungi continues to be a challenge in the clinical laboratory. However, with the advent of more effective immosuppressant therapy, increasing age of the population, and the continuing struggle against the HIV/AIDS epidemic, opportunistic fungal infections are increasing. We have developed a simple, PCR-based assay, capable of identifying any fungus from culture in about 12 hours in any clinical molecular lab.

Design: Fungi were cultured with $0.5 \mathrm{~cm}$ fragments of hyphal mats or colonies of yeast picked and frozen at $-20 \mathrm{C}$. Fungal colonies were resuspended using grinding as necessary in $500 \mathrm{ul}$ of $95 \mathrm{C}$ lysis buffer (Ambion Recover-All nucleic acid kit) and incubated for 30 minutes. Fungal DNA was extracted from 50 ul resuspended fungi using the buffers and protocol of the Qiagen FFPE DNA extraction kit. The protocol was modified to remove the de-paraffinization steps. PCR was performed using modified primers ITS1-5'-TCCGTAGGTGAACCTGCGG-3' and ITS2-5'GCTGCGTTCTTCATCGATGC-3' that amplify the ITS1 region in all fungi. PCR products were assessed on $1.5 \%$ agarose-TBE gels and purified from gel using Qiagen gel extraction kits. DNA was sequenced using BigDye and used to interrogate the BLAST database.

Results: Sixteen out of sixteen (100\%) fungi of known species including Aspergillus terreus, A niger, A nidulans, Candida albicans, C. parapsilosis, C kruzei, C. tropicalis, C. glabrata, Cryptococcus neoformans, C. laurentii, Epicoccum nigrum, Exserohilum rostrate, Fusarium solani, Pithomyces chartarum, Rhizomucor variabilis, and Rhizopus microsporus were identified to species with $94-100 \%$ sequence identity and E-values from $4.00 \mathrm{E}-22$ to $3.00 \mathrm{E}-175$. Of the 33 live clinical isolates coming from lung, skin, nail, wound, or incidental culture, 31 were identified (94\%). Of these, 26 had a laboratory diagnosis and there were three substantial discrepancies ( $88 \%$ accuracy). The seven remaining sequence-identified fungi were unidentified in the lab as sterile hyphae or molds.

Conclusions: We have developed a high-accuracy PCR-based fungal identification assay that can be performed with minimal reagents and minimal capital expense using tools, techniques, and reagents common to any molecular pathology laboratory. While this assay has significantly better accuracy and sensitivity to many other lab-derived assays in the literature, the small but not insignificant number of inconsistencies mean clinical and morphological criteria still must be part of the final diagnosis. In cases where a morphologic identification cannot be made, this assay has potential to augment current practice.

1609 Emerging Pattern of Helicobacter pylori Clarithromycin Resistance in the United States

JY Park, M Mitui, CA Arnold, M Gallegos, E Coss, B Cryer, D Lam-Himlin, NKLeos, CD Doern. UT Southwestern Medical Center, Dallas, TX; Children's Medical Center, Dallas, TX; Ohio State University, Columbus, OH; Mayo Clinic in Arizona, Scottsdale, AZ. Background: Helicobacter pylori (HP) is a significant cause of gastritis and the development of gastric malignancies. Clarithromycin (CLR) is a major antibiotic for the empiric treatment of HP gastritis. CLR should not be used empirically when local CLR resistance is $>20 \%$. Globally, CLR resistance has risen over the past decade. The last survey of HP CLR resistance in the United States (US) was from the 1990s and had no geographic region with $>15 \%$ resistance. The current study surveyed CLR resistance in multiple institutions across the US.

Design: Formalin-fixed paraffin embedded (FFPE) gastric biopsies with a diagnosis of HP gastritis were retrospectively obtained from four tertiary care institutions from the Midwest $(n=1)$, South $(n=2)$ and West $(n=1)$ US Census designated regions. Specimens were obtained from 2011 to 2013, and were chronologically consecutive cases of HP gastritis within each institution. FFPE specimens were sequenced for HP 23S rRNA mutations associated with CLR resistance.

Results: FFPE specimens were obtained from 103 patients (Midwest $=25$, South $=26$ and 28 , West $=24$ ). Patients had a median age of $60 \mathrm{y}$ (range 22 to 87 ) and $59 \%$ were female. Patient self-identified race was $55 \%$ Caucasian $(n=57), 22 \%$ African-American $(n=23), 14 \%$ Hispanic $(n=14), 9 \%$ other $(n=9)$. The overall rate of CLR resistance was $26 \%$. By race, the resistance rates were Caucasian $=33 \%$, African-American $=23 \%$, Hispanic $=14.3 \%$, other $=44 \%$. By region, the resistance rates were Midwest $=40 \%$, South $=23 \%$ and $0 \%$, West $=46 \%$.

Conclusions: In this study, only one institution had a resistance rate low enough $(0 \%)$ to allow for empiric CLR therapy. The resistance rate at the remaining institutions and the overall resistance rate exceed the $20 \%$ threshold for empiric therapy. The number of specimens and sites in this study is small, but indicates the possibility of increased in CLR resistance compared to previous US surveys. The use of standard empiric HP antibiotic regimens based on CLR needs to be promptly re-evaluated in the US. The methodology of this study is based on DNA sequencing of FFPE tissue; this opens the possibility of performing rapid HP epidemiologic studies on residual clinical specimens.

1610 Routine Testing for Anaerobic Bacteria in Cerebrospinal Fluid Cultures Improves Recovery of Clinically Significant Pathogens

ME Pittman, BS Thomas, MA Wallace, CJ Weber, C-AD Burnham. Washington University, St Louis, MO.

Background: Although widespread use of vaccines targeting Haemophilus influenzae and Neisseria meningitidis have dramatically altered the epidemiology of bacterial meningitis, standard methods for culturing cerebrospinal fluid (CSF) specimens have not changed. As there are now increasing numbers of patients with central nervous system (CNS) hardware and associated reports of unexpected anaerobic bacterial meningitis, the purpose of this study was to assess the clinical utility of routinely querying for anaerobes in CSF cultures.

Design: Brucella blood agar (BBA) incubated anaerobically was added to the standard laboratory work-up of all CSF cultures. Data were analyzed from positive cultures submitted in the 12 months prior to $(n=49 / 2352)$ and following $(n=99 / 2302)$ the addition of BBA. Electronic medical records were reviewed for patient demographics, history, culture results, and outcome. Clinical significance of the organism recovered was assessed in two ways: if the patient received therapy for the organism, and if an infectious diseases specialist in blinded case review would have recommended therapy. Results: Patient demographics and presenting symptoms were similar in the pre- and post-BBA years, with the majority of patients presenting with altered mental status (51\%). Overall, 34 anaerobic bacteria were cultured (23\%), two pre-BBA $(4 \%)$ and 32 post-BBA $(32 \% ; p=0.01)$. The anaerobic organisms isolated were Propionibacterium species $(\mathrm{n}=15)$ and $P$. acnes $(\mathrm{n}=18)$, with one Bacterioides thetaiotaomicron. Two $P$. acnes cultures had rare growth on aerobic media, but all anaerobes grew on BBA. Anaerobic isolates were just as likely as aerobic isolates to be from immunosuppressed patients $(p=0.07)$ or patients with CNS hardware $(p=0.67)$, but they were less likely to have findings on specimen Gram stain $(p=0.04)$. Treatment was given to $54 \%$ of patients with positive aerobic cultures and $24 \%$ with positive anaerobic cultures $(p=0.002)$. The correlation between prospective and retrospective assessment of clinical significance was excellent $(\kappa=0.94)$. Of the 8 patients with anaerobic organisms who received treatment, 6 had CNS hardware, one had known spinal osteomyelitis, and one had an intracranial malignancy.

Conclusions: Anaerobic bacteria are a cause of clinically significant bacterial meningitis in the post-vaccine era. The addition of a single piece of anaerobic media to standard CSF cultures improves detection of these organisms and allows for the timely initiation of antimicrobial therapy.

1611 Surgical Pathology of Skeletal Coccidioidomycosis: A MultiInstitutional Clinical and Histologic Analysis of 25 Cases (2000-2012)

$R W$ Ricciotti, TA Shekhel, JE Blair, TV Colby, RE Sobonya, BT Larsen. University of Arizona, Tucson, AZ; Mayo Clinic, Scottsdale, AZ; Mayo Clinic, Rochester, MN. Background: Skeletal coccidioidomycosis is rare, and has not been extensively studied. Design: All skeletal coccidioidomycosis cases (2000-2012) requiring surgical intervention were retrieved from the pathology files of the University of Arizona and Mayo Clinic in Arizona. Clinical history and imaging were reviewed. Spherule density was measured and histologic features were scored $(0-3+)$.

Results: Among 25 patients (median age 41 yrs; 17 men), infections involved bones ( 2 cases), joints (6), or both (17). Ethnic minorities were disproportionately represented (12). History included previously documented coccidioidomycosis (13), diabetes (6), leukemia (2), autoimmune diseases including sarcoidosis (3), rheumatoid arthritis (3), and other disorders (3), and iatrogenic immunosuppression with steroids (8), methotrexate (2), and other medications (3). Symptoms (median 3 months) included pain/arthralgia (21), swelling (10), fever (2), and malaise (1). Sites included the wrist/ hand (8), pelvis (5), ankle/foot (3), knee (3), elbow (2), spine (2), tibia (1), and skull (1). Imaging showed destructive bone lesions (17), synovial/soft tissue involvement (12), and joint effusion (2). Cultures and serology were positive in 15 of $17(88 \%)$ and 19 of 22 patients $(86 \%)$, respectively. Treatment included surgical debridement(s) and antifungal medications. Histologic review of 27 debridements showed granulomatous inflammation in all cases, with granulomas ranging from poorly to well formed, with 
or without necrosis. Spherule density varied (mean $4.8 / \mathrm{hpf}$; range $<0.1$ to $13.5 / \mathrm{hpf}$ ) and was higher in immunodeficient patients (median 4.3/hpf vs. 1.6/hpf). Mean scores for all inflammatory cell types were lower in the immunodeficient group, while necrosis and fibrosis were higher. Eosinophils were usually rare. 12 patients experienced recurrent infections, but all 25 improved with treatment.

Conclusions: The distal extremities are the most common sites of skeletal coccidioidomycosis requiring surgical intervention. This condition is strongly associated with autoimmune disorders and immunosuppression. Histologic features vary and depend on immune status, with immunocompromised patients showing less inflammation and greater spherule numbers, necrosis, and fibrosis. Spherules may be rare, particularly in immunocompetent patients, and multiple modalities including serology, culture, and histology may be required for diagnosis.

1612 Diagnosis of Cutaneous Leishmaniasis: Why Punch When You Can Scrape?

MW Saab, R Hoteit, D Shammaa, F Abbas, R Mahfouz, I Khalifeh. American University of Beirut Medical Center, Beirut, Lebanon.

Background: Cutaneous Leishmania (CL) has been introduced to the Leishmania-naive Lebanese population in an uncontrolled manner due to recent large scale displacement of Syrian refugees from regions endemic to CL. Accordingly, a quick and reliable method to diagnose CL and initiate treatment is essential to avoid an impending epidemic.

Design: From $133 \mathrm{CL}$ patients, we obtained matched biopsies (bx) and air-dried scrapings in 72 patients [male/ female: 33/39; mean age 18 years (range: 1-61)] Scrapings were collected in 2 forms: thick drop $(n=33)$ and thin smears $(n=39)$. Age, sex, lesion duration, anatomic location, number and type (wet or dry) of lesions were collected. For microscopic evaluation, biopsy sections stained with H\&E and scrapings stained with Wright Giemsa, Diff Quick, Papanicolau and H\&E, were examined. Parasitic index (PI) was recorded. PCR was performed on both scrapings and biopsies using Leishmania-specific ribosomal internal transcribed spacer 1 (ITS1-PCR), followed by digestion of the ITS1-PCR amplicons with HaeIII for subsequent restriction fragment length polymorphism (RFLP) speciation.

Results: CL lesion duration varied from 0.5 to 27 (mean: 5.8 ) months and were located in head/neck (39/72) area, upper (31/72) and lower extremities (18/72). The diagnostic sensitivity of each test performed revealed that microscopy in conjunction with PCR on punch biopsies was the most sensitive test (93\%) overall. However, taken individually, microscopy and PCR yielded the highest sensitivities when performed on drop scrapings (63\% and $85 \%$, respectively), and not smear scrapings ( $38 \%$ and $56 \%$, respectively) as compared to the punch biopsies ( $44 \%$ and $83 \%$, respectively)

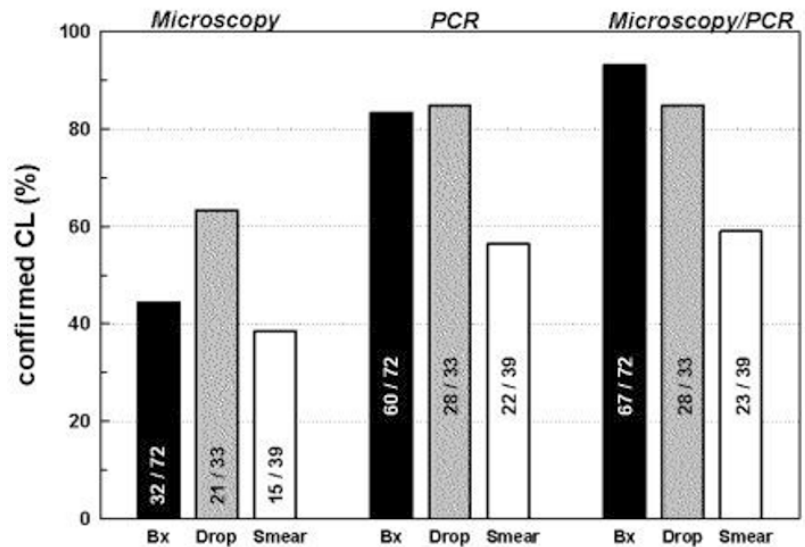

Microscopic concordance for punch biopsies and drop scrapings was present in 25 of 33 cases versus 8 discordant findings. Agreement/disagreement for these two techniques was predicted only by high/low parasitic index (PI: $3.1 \pm 1.7$ and $0.4 \pm 0.5$, respectively; $\mathrm{p}<0.05$ )

Conclusions: We optimized a novel rapid method for reliable diagnosis of CL based on "drop scrapings" with good agreement with the gold standard "punch biopsy" technique. We accordingly propose a practical algorithm to confirm CL based on initial "drop" microscopy followed by PCR and later biopsy as necessary.

1613 A New Face of Cutaneous Leishmaniasis in a Time of War M Saroufim, K Charafeddine, I Khalifeh. American University of Beirut, Beirut, Lebanon.

Background: Cutaneous leishmaniasis (CL), a potentially chronic and disfiguring condition, has been thrust in to the spotlight following reports among military personnel returning from the Near East. Lebanon (LB), a non-endemic area, is now suffering a health care crisis in the wake of a CL epidemic brought from endemic Syria by the protracted conflict in the region, resulting in the displacement of over $1,000,000$ refugees into LB.

Design: Punch biopsies ( 1 patient sampled/displaced family, $n=110$ ) were taken for histologic examination (parasitic index) and molecular speciation by PCR. Demographics, migration patterns, lesion number and characteristics including presence of extensive disease (ED) were documented. ED was defined as having $\geq 1$ of the following: Disfiguring, threatening the function of vital sensory organs, lesion present for $>12$ months, $>3 \mathrm{~cm}, \geq 5$ lesions and special forms of CL (i.e. sporotrichoid). Results: The refugees fled from endemic and non-endemic areas alike, had been in LB 5 months on average and $77 \%$ of them reported the appearance of the first lesion after being in LB for $>2$ months (average incubation period 2-8 weeks). PCR resulted in 94 cases of L. tropica and 16 L. major types. In this special conflict population, the preponderance of patients sampled were under 18 years old (66/110 patients) and an average of $52 \%$ members were affected/family (mean number of members $=6$ ). The majority of patients met criteria for $\mathrm{ED}(\mathrm{n}=65,59 \%)$ including: 30 with disease compromising a sensory organ, 10 special forms, 41 disfiguring, $54>3 \mathrm{~cm}$ and $10 \mathrm{chronic}$ lesions. Parasitic index, molecular subtype and geographic location were similar for ED versus non-ED group. ED was more prevalent among children (median 9 vs. 21 years; $p=0.002$ ) and was more frequently observed on the face and lower extremities $(\mathrm{p}=0.002)$. Both age and anatomic location were predictors of ED by multivariate logistic regression.

Conclusions: In studying this epidemic, we are seeing a new face of CL in times of war; stressful and unsanitary living conditions may account for the uncharacteristically high number patients with ED.

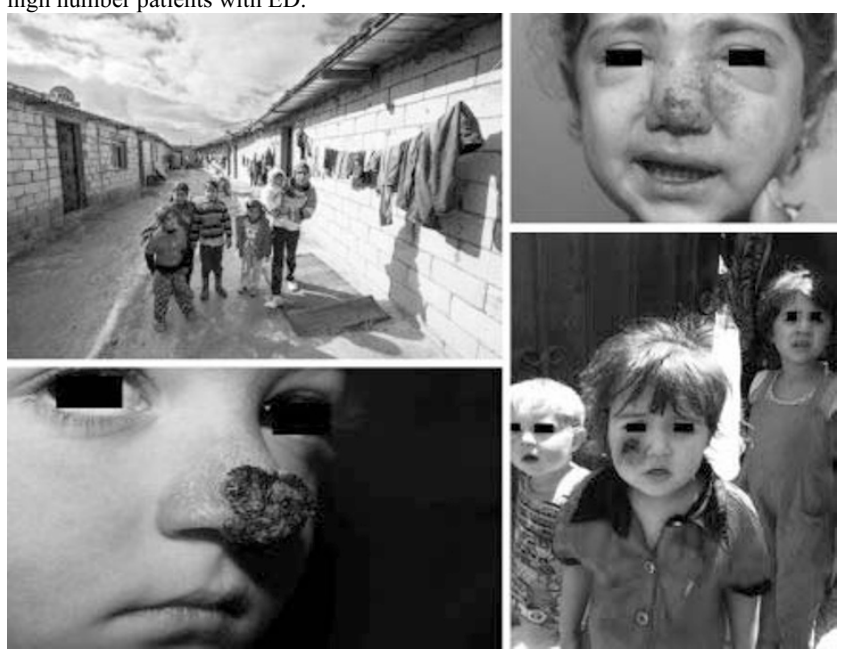

Furthermore, the majority of patients reported appearance of lesions well beyond average documented incubation periods suggesting the transfer and propagation of CL to LB and other non-endemic countries harboring refugees.

1614 Disparities in Clinicopathological Features of Anal Squamous Cell Carcinoma (ASCC) in HIV+ and HIV- Patients: A Retrospective Study in an Inner City Hospital

S Seydafkan, G Johnson, S Ehdaivand, M Mosunjac. Emory, Atlanta, GA.

Background: ASCC has become one of the most common non-AIDS defining malignancies in the era of HAART. Due to the lack of uniform screening and treatment guidelines for ASCC, there is no consistent approach to these patients. The differences, in the natural history of ASCC in HIV+ and HIV -patients has not been well elucidated. In this study, we aimed to analyze possible demographic and clinicopathological differences in HIV + and HIV- patients with ASCC.

Design: In a retrospective search (1999-2013), 37 cases of ASCC were identified. Electronic files were used for extraction of demographic and laboratory data, pathology reports and types of treatment.

Results: Of the 37 ASCC patients, there were $20 \mathrm{HIV}+(54 \%)$ and $17 \mathrm{HIV}-(46 \%)$ There were a total of 9 females and none were HIV+. Less than $1 / 3$ of males were HIV-. table1

\begin{tabular}{|c|c|c|c|}
\hline & MALE & FEMALE & TOTAL \\
\hline HIV + & $20(71.42 \%)$ & 0 & $20(54.05 \%)$ \\
\hline HIV - & $8(28.57 \%)$ & $9(100 \%)$ & $17(45.94 \%)$ \\
\hline TOTAL & $28(75.67)$ & $9(24.32 \%)$ & 37 \\
\hline
\end{tabular}

Women were on average 10 years older $(54+10.6)$ than men $(45+8.5)$ and HIV$(52.4 \pm 9.4)$ patients were 10 years older than $\mathrm{HIV}+(43 \pm 8)$. Overall, less than $1 / 2$ of the patients presented with early stage disease, only $22 \%$ presented with stage I. More than $1 / 2$ of the patients presented with stage III or greater stage $(54 \%)$. No HIV + patients presented with metastatic disease while $18 \%$ HIV- patients had distant metastasis at the time of diagnosis.[table2]Most of the HIV+ patients were on HAART (80\%), and $41 \%$ fulfilled the criteria for AIDS. All except one of the females had negative cervical pap and none of them had anal smears (AS). Only 7 male HIV+ patients had AS before the diagnosis of ASCC; all of the AS were atypical but none were flagged as invasive. $67 \%$ of patients received radiation therapy, but majority were lost to long term follow up. Conclusions: In our inner city hospital, ASCC in the HIV+ population afflicted younger males compared to HIV -, who tended to be older females. HIV+ patients tended to have locally advanced disease confined to the anogenital area whereas HIV-tended to have metastasis at the time of diagnosis. These data raise the possibility that there may be slightly different pathobiology in HIV+ vs. HIV-ASCC. There is no significant difference in initial stage of the disease and HIV status. As there are no guidelines for anal cancer screening, only a small percentage of patients had AS, but all of them had abnormal findings. This might suggest the need for a more aggressive screening approach in the high risk population, recognizing that an important, less common group exists that may have no risk factors. 
1615

Type I Interferon Signaling Mediates Toxic Shock-Like Syndrome Induced by Intracellular Bacteria

HL Stevenson, Q Yang, M Scott, N Ismail. UPMC, Pittsburgh, PA.

Background: Ehrlichia chaffeensis is an obligately intracellular bacterium that causes fatality by inducing toxic shock-like syndrome and provides an excellent model of immune-mediated liver pathology. The liver is the primary site of pathology and infection results in extensive hepatocyte necrosis and apoptosis, which is associated with over activation of the immune response including secretion of inflammasomedependent IL- $1 \beta$ and IL- 18 , and expression of IFN $\alpha / \beta$. Although selectively inhibiting the main pathogenic immune cells such as neutrophils, $\mathrm{NK}$, and $\mathrm{CD} 8^{+} \mathrm{T}$ cells is partially protective resulting in prolonged survival and decreased pathology, the key event that leads to over activation of immunity is not known.

Design: Although type 1 interferons are well known for providing critical defense against viruses and bacteria, they have recently been shown to promote sepsis. We hypothesized that activation of the inflammasome pathway by NLRP3, caspase 1, and/or type 1 IFN may be the key event leading to immunopathology in our model of toxic shock-like syndrome. We addressed the role of type 1 interferons by examining Ehrlichia infection of $\mathrm{C} 57 \mathrm{BL} / 6$ mice lacking type 1 IFN receptors (IFN $\alpha / \beta \mathrm{R}^{-/}$).

Results: Surprisingly, infected IFN $\alpha / \beta \mathrm{R}^{-/}$mice were completely protected against a normally lethal dose of bacteria and survived past 30 days post-infection. Protection of the IFN $\alpha / \beta \mathrm{R}^{-/}$mice correlated with effective bacterial clearance, altered pathology, increased IFN- $\gamma$ in sera, decreased IL-10 in sera and spleens, and increased frequency of protective $\mathrm{CD}^{+} \mathrm{Th} 1$ and NKT cells in the spleen. Furthermore, lack of IFN $\alpha / \beta R$ signaling significantly abrogated production of IL-1 $\beta$, suggesting that inflammasome activation during lethal disease is linked to IFN $\alpha / \beta$ signaling. To test this possibility, we infected NLRP $3^{-}$and Caspase $1^{-/}$mice with Ehrlichia. Compared to complete protection of IFN $\alpha / \beta R^{-/}$mice, NLRP3 ${ }^{-}$and Caspase $1 /$ mice were only partially protected. These mice also had lower bacterial burdens, attenuated hepatic necrosis and apoptosis, and decreased expansion of neutrophils. Interestingly, production of type I IFN was lower in NLRP3 $\%$ and Caspase $1 \%$ mice than in WT mice, but was not completely abrogated. The latter could account for incomplete protection of these mice, and suggests that type 1 IFN signaling plays a major pathogenic role upstream of the NLRP3 inflammasome. Conclusions: IFN $\alpha / \beta$ signaling is a master regulator of fatal intracellular bacteriamediated toxic shock-like syndrome and warrants further investigation in human sepsis-like syndromes.

\section{Informatics}

1616 Regulatory Genomics of the Kidney Illuminated by DNasel-seq $S$ Akilesh, JA Stamatoyannopoulos. University of Washington, Seattle, WA.

Background: A fundamental role for epigenomic defects and altered transcriptional regulatory programs in the pathogenesis and clinical biology of both neoplastic and non-neoplastic disease is increasingly evident. However, we still lack basic knowledge of the functional genomic architecture of major tissues, creating a major obstacle to systems-level approaches for understanding and treating disease. New technologies now allow us to surmount this bottleneck to efficiently map regulatory DNA (e.g. promoters, enhancers, insulators) on a genomic scale.

Design: Regulatory DNA is characterized by the cooperative binding of sequencespecific transcriptional regulatory factors in place of a canonical nucleosome, leading to an altered chromatin state that is characterized by markedly heightened accessibility to nucleases such as DNaseI. DNaseI treatment followed by high throughput sequencing was previously applied to kidney tissue ( $n=65$ from day $82-147$ fetuses) and the resulting datasets were deposited in the NIH Roadmap Epigenomics Mapping Consortium (NREMC) database. We are applying novel bioinformatic analyses developed for the ENCODE project ( $\sim 30$ papers Nature,Cell, Science, 2012$)$ to understand the changing regulatory landscape of the kidney during development. Results: Preliminary analysis of fetal kidney datasets reveals a wide range of DNaseI hypersenitive sites (DHS) in fetal kidney tissues (range 128,051-247,500, Fig. 1).

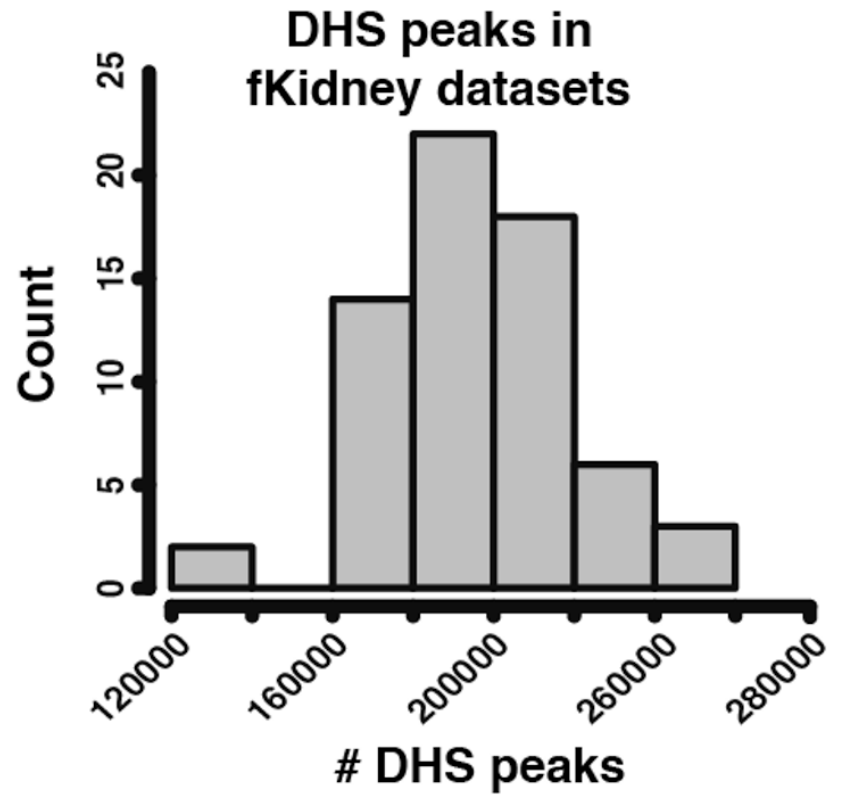

\# DHS peaks

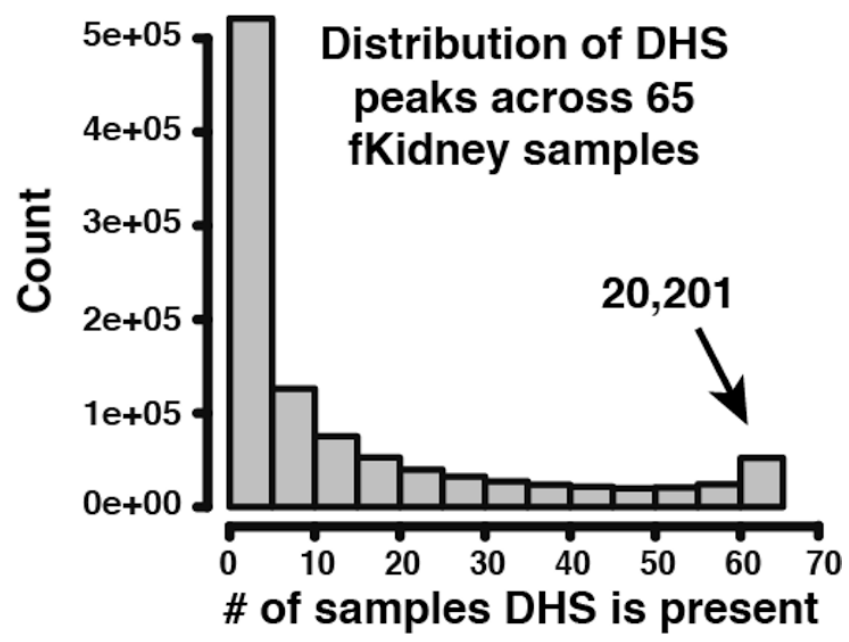

While many DHS are present only in a few samples, a set of 20,201 DHS are present in all fetal kidney tissues analyzed (Fig. 2). Further evaluation of these conserved and differentially expressed DHS is in progress to mine the regulatory DNA landscape of the kidney.

Conclusions: Global DHS profiling using DNaseI treatment and next-generation sequencing technologies will provide novel insights into the changing regulatory DNA landscape of the kidney.

1617 Synoptic Report Generators for Hematopathology: Development of a System Embedded within the Anatomic Pathology Laboratory Information System (APLIS) That Integrates Morphology, Flow Cytometry, Immunohistochemistry and Molecular Genetics

A Alkhasawneh, JR Ross, CM Carter, SZ Al-Quran, Y Li, RW Allan. University of Florida, Gainesville, FL.

Background: Synoptic reporting for cancer specimens have increased the consistency, completeness of reporting, ease of interpretation and creating of discrete value reporting that allows for ease in data mining. APLIS systems now contain embedded synoptic reporters for cancer cases, but such systems do not handle the complex reporting of hematopathology specimens and do not allow for synoptic reporting of morphology, flow cytometric, immunohistochemical and molecular genetic findings. We sought to develop an embedded custom synoptic report generator for transplantation pathology specimens using our anatomic pathology laboratory information system(APLIS) (PowerPath, SunQuest).

Design: We developed custom synoptic reporting- based embedded templates using the worksheet function within our APLIS (PowerPath) for hematopathology specimens. The programming is easy to perform and using standard choice fields: drag and drop, multiple choice, complex choice and free text reporting options. These synoptic checklists included pertinent morphologic findings (degree of dysplasia, qualitative assesment of lineage maturation, blast counts), flow cytometric findings (antibody and corresponding intensity), immunohistochemistry (antibody performed, qualitative and quantitative result for certain antibodies such as CD34, CD138). In addition, karyotype 